\title{
Influência do ciclo lunar na atividade de vôo de Coquillettidia (Rhynchotaenia) venezuelensis (Theobald) (Diptera, Culicidae) na Mata Atlântica, Serra do Marumbi, Morretes, Paraná, Brasil ${ }^{1}$
}

\author{
Adson Luís Sant'Ana ${ }^{2}$ \\ Ana Leuch Lozovei ${ }^{3}$
}

\begin{abstract}
Influence of the Lunar Cycle on the Flight Activity of Coquillettidia (Rhynchotaenia) venezuelensis (Theobald) (Diptera, Culicidae) in the Atlantic Forest, Marumbi mountain, Morretes, Paraná State, Brazil. From October 1995 to January 1996 a survey of the culicid species mentioned above was carried out in a restrict Atlantic Forest area, located in the Marumbi mountain (approximately 6,5 km from the town of Morretes). Shannon light trap was used during 48 nights, in the four lunar phases: full moon, first quarter, new moon and last quarter between evening and morning twilights. A total of 594 Coquillettidia (Rhynchotaenia) venezuelensis (Theobald, 1912) specimens were collected and the different night flight activity in the four moon phases was observed. Regarding the hourly activity, the peak higher numbers of Coquillettidia venezuelensis were collected in the first three hours. Concerning the environmental factors, temperature was found to be the more relevant one for the flight activity of Coquillettidia venezuelensis.

KEY WORDS. Culicidae, Coquillettidia venezuelensis, fly activity, four moon phases
\end{abstract}

Em diferentes partes do mundo, houve pesquisas sobre a influência da luminosidade lunar na atividade de vôo de mosquitos. Alguns estudos evidenciaram que a atividade é intensificada durante a lua nova, quando comparada com as noites de lua cheia (BRADLEY \& MCNEEL 1935, coletando várias espécies de mosquitos; Hosfall 1943, capturando espécies de Anopheles, veiculadoras de malária). ProVOST (1959), trabalhando com algumas espécies de mosquitos e FOLEY et al. (1992), investigando Anopheles (Cellia) annulipes Walker, 1856, ao contrário, chegaram à conclusão de que a atividade de vôo destes mosquitos é mais intensa durante a lua cheia.

As pesquisas envolvendo este assunto também foram desenvolvidas por vários autores. BIDLINGMAYER $(1964,1974)$, no primeiro trabalho, estudou o efeito da luminosidade lunar sobre a atividade de vôo de diversas espécies de mosquitos, enquanto no segundo, a influência de fatores ambientais e estágios fisiológicos sobre

1) Contribuição número 1243 do Departamento de Zoologia, Universidade Federal do Paraná

2) Departamento de Parasitologia, Universidade Federal de Minas Gerais. Avenida Presidente Antônio Carlos 6627, Pampulha, 31270-901 Belo Horizonte, Minas Gerais, Brasil.

E-mail: asantana@mono.icb.ufmg.br

3) Departamento de Patologia Básica, Setor de Ciências Biológicas, Universidade Federal do Paraná. Caixa Postal 19031, 81531-990 Curitiba, Paraná, Brasil.

E-mail: alozovei@bio.ufpr.br 
os padrões de vôo de mosquitos em captura com armadilha luminosa; DAVIES (1975) verificou a atividade de hematofagia de duas espécies de Culex (Melanoconion) durante as fases lunares; CHARLwOOD et al. (1986) conferiram a influência da luminosidade lunar sobre a atividade de picadas hematofágicas de Anopheles (Cellia) farauti Laveran, 1902; Mishira et al. (1996) e SiNGH et al. (1996) verificaram, respectivamente, a influência da luminosidade lunar em capturas com armadilha luminosa e a influência das fases lunares sobre a captura de Anopheles (Cellia) culicifacies Giles, 1901.

Nas Américas, poucos trabalhos foram produzidos com referência à intensidade luminosa das quatro fases lunares. Dentre eles, destacam-se o de PRATT (1948), em Porto Rico, em que utilizando a armadilha luminosa New Jersey estudou Anopheles (Nyssorhynchus) albimanus Wiedemann, 1820 e o de RUBIO-PALIS (1992), na Venezuela, que conduziu um estudo sobre Anopheles (Nyssorhynchus) nuneztovari Gabaldón, 1940 com auxílio de armadilha luminosa CDC durante as quatro fases lunares.

O presente trabalho foi desenvolvido com Coquillettidia (Rhynchotaenia) venezuelensis (Theobald, 1912), um vetor potencial de agentes de arboviroses, tendo sido encontrado naturalmente infectado com esses agentes, inclusive com os de Oropouche (FORATTINI 1965b; HERVÉ et al. 1986). Esta espécie, ao proliferar em criadouros próximos a residências, torna-se muito incômoda aos habitantes devido a picadas agressivas infligidas aos humanos e aos animais domésticos, tanto de dia como à noite (CONSOLI \& LOURENÇO-DE-OLIVEIRA 1994).

A presente investigação, durante as quatro fases do ciclo lunar, teve por objetivo verificar se a atividade noturna de vôo de Coquillettidia venezuelensis é realmente influenciada pelas diferentes intensidades luminosas lunares, em contraposição à fonte luminosa fluorescente de intensidade constante em torno de 70 lux, nas paredes da armadilha de Shannon e, ao mesmo tempo, detectar a freqüência horária da atividade do crepúsculo vespertino ao crepúsculo matutino e, ainda, avaliar a influência das variáveis ambientais de temperatura, umidade relativa do ar e pluviosidade. São dados importantes para os conhecimentos da espécie em questão, que tem importância epidemiológica.

\section{MATERIAL E MÉTODOS}

As coletas de Coquillettidia venezuelensis foram realizadas na área litorânea do Estado do Paraná, a 6,5 km a noroeste da cidade de Morretes e a $3 \mathrm{~km}$ ao sul do

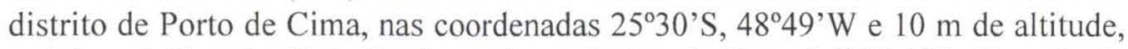
próximo à Estação II do Instituto Agronômico do Paraná (IAPAR), Estrada da Graciosa. A área de estudo fica localizada no sopé da Serra do Marumbi, com densa floresta ombrófila das terras baixas preservada (VELoso et al. 1991). A Serra do Marumbi destaca-se por cumes mais altos da região, com o Pico Marumbi de 1.547 $\mathrm{m}$ e o Morro do Leão de $1.564 \mathrm{~m}$ de altitude.

A pesquisa desenvolveu-se de 07 de outubro de 1995 a 28 de janeiro de 1996 por três noites consecutivas em cada uma das quatro fases do ciclo lunar. Em consequêencia, isto envolveu quatro ciclos lunares de intensidade luminosa. Foram 
no total 48 noites trabalhadas durante quatro meses para obter amostras de mosquitos, cabendo 12 noites para cada fase lunar: lua cheia, quarto minguante, quarto crescente e lua nova. Utilizou-se uma armadilha de Shannon com fonte luminosa fluorescente de $20 \mathrm{~W}$ ligada a uma bateria de $52 \mathrm{~A}$. Antes de cada coleta, a carga da bateria era restabelecida por um carregador de baterias doméstico.

A armadilha sempre foi instalada uma hora antes do crepúsculo vespertino e retirada uma hora depois do matutino, calculados conforme tabela de horários dos "Almanaques náuticos" (DHN 1995, 1996). Estabeleceu-se, portanto, sete intervalos horários com duração de duas horas cada, para em cada um deles apanhar com aspirador os mosquitos no interior da armadilha. Estes horários ficaram assim organizados: Hora 1, crepúsculo vespertino e uma parte da noite; Horas 2 a 6 , todas compreendendo só parte da noite, enquanto a última, a Hora 7 , crepúsculo matutino e uma parte do dia seguinte. As horas I a 4 iam do crepúsculo vespertino e noite adentro até cerca da meia-noite, enquanto as horas 5 a 7 , da meia-noite em diante até o fim do crepúsculo matutino. Este procedimento foi necessário para adequar o arranjo dos crepúsculos.

Os mosquitos foram recolhidos da armadilha com um aspirador manual movido a 8 pilhas durante os quinze minutos finais de cada intervalo horário. Neste tempo, foram anotados os dados da temperatura ambiente e da umidade relativa do ar conferidos com um termômetro-higrômetro, ao passo que com pluviômetro e proveta volumétrica padrão foi determinada a quantidade de chuva no próprio local da pesquisa. A constância da intensidade luminosa (lux) emitida pela lâmpada fluorescente foi monitorada com luxímetro portátil alimentado por bateria.

Os mosquitos foram triados ao microscópio estereoscópico e montados em alfinetes entomológicos. Para identificar as espécies, serviu-se de chaves taxonômicas (FoRATTINI 1965a,b) e de auxílio de especialistas. As abreviaturas de gêneros seguiram as normas de REINERT (1975). Alguns exemplares de mosquitos foram depositados no acervo do Museu de Entomologia "Padre Jesus Santiago Moure", Universidade Federal do Paraná, (DZUP).

Para verificar a significância ou não da influência de algumas variáveis nos resultados obtidos, foram aplicados testes estatísticos não-paramétricos (SIEGEL 1975), como o teste de Friedman ANOVA e Kendall, comparações múltiplas em caso de a hipótese Ho ser rejeitada pelo teste ANOva e ainda Wilcoxon Matched Pairs Test. Igualmente, foi feito teste de regressão para os dados de temperatura ambiente.

Para o teste de Friedman ANOVA, foram estabelecidas as hipóteses:

Ho: Não existe diferença significativa entre as variáveis analisadas.

H1: Existe pelo menos uma diferença entre as variáveis analisadas.

Caso a hipótese Ho tivesse sido rejeitada pelo teste de Friedman ANOVA, realizaram-se comparações múltiplas para detectar onde estava a diferença.

Para o Wilcoxon Matched Pairs Test, estabeleceu-se as hipóteses:

Ho: hora $1+$ hora $2+$ hora $3=$ hora $4+$ hora $5+$ hora $6+$ hora 7

$\mathrm{H} 1$ : hora $1+$ hora $2+$ hora 3 \# hora $4+$ hora $5+$ hora $6+$ hora 7 


\section{RESULTADOS E DISCUSSÃO}

A atividade de vôo de Coquillettidia venezuelensis em todas as fases lunares, registrada em ordem decrescente, apresentou os seguintes valores: 218 exemplares (117 fềmeas e 101 machos) na lua crescente, 141 (106 fêmeas e 35 machos) na lua cheia, 124 ( 89 fêmeas e 35 machos) na lua minguante e, finalmente, 112 (88 fềmeas e 24 machos) na lua nova (Tab. I, Fig. 1). Pelo teste de Friedman ANOVA, a hipótese Ho foi aceita ( $\mathrm{P}<0,5794)$. Estatisticamente, não existe significância no número de espécimes de Coquillettidia venezuelensis nas fases lunares.

No conjunto das quatro fases lunares, a freqüência horária deu-se em números mais elevados, tanto na soma de ambos os sexos como na de sexos separados, nos primeiros quatro intervalos horários, ou seja, do intervalo horário 1, que inclui o início do crepúsculo vespertino até o intervalo horário 4 , um pouco antes da meia-noite. (Tabs I, II; Fig. 2). Portanto, a atividade mais intensa de vôo de Coquillettidia venezuelensis nas quatro fases lunares aconteceu do crepúsculo vespertino até perto da meia-noite. Da meia-noite em diante, o número de espécimes diminuiu drasticamente, chegando a pequenos valores e à total ausência (Tab. I). Aplicando o teste de Friedman ANOVA, a hipótese Ho foi rejeitada. Pela comparação múltipla, a hipótese Ho foi rejeitada entre os intervalos horários 1 e 6,1 e 7,2 e 4,2 e 5, 2 e 6,2 e 7, 3 e 6,3 e 7, 4 e 6 e ainda 6 e 7 ao nível de significância de $<0,1 \%$.

BIDLINGMAYER (1964) salienta que os efeitos diretos da luminosidade lunar sobre a atividade de vôo de insetos não têm sido extensivamente estudados a tal ponto que se possa inferir quanto aos efeitos indiretos desta sobre as capturas de mosquitos com armadilhas luminosas. Vários trabalhos têm revelado que as capturas são mais reduzidas durante o período de lua cheia em contraste com o período de lua nova. A intensidade luminosa constitui um fator muito importante para atividade de vôo dos mosquitos, conforme relatam BRADLEY \& MCNEEL (1935), Hosfall (1943), Pratt (1948), Provost (1959), Bowden (1973), Rubio-Palis (1992) e FOLEY et al. (1992). Salientam os pesquisadores que este efeito deve-se à menor eficiência física da armadilha luminosa durante o período de lua cheia competindo com a luminosidade lunar, em conseqüência do que exerce menor atratividade. Entretanto, alguns trabalhos revelam que muitos mosquitos noturnos são mais ativos durante a lua cheia, quando comparado com períodos de menor intensidade da luminosidade lunar (BIDLINGMAYER 1964; DAVIES 1975; CHARLWOOD et al. 1986; CHADEE 1992), sugerindo que na lua cheia haja um prolongamento da intensidade luminosa e esta condição favoreça a habilidade dos mosquitos para localizar hospedeiros e sítios de oviposição (ALLEN et al. 1987). LOURENÇO-DE-OLIVEIRA (1985) em sua pesquisa constatou que houve maior quantidade total de mosquitos durante a lua minguante. Na presente investigação, a espécie estudada apresentou maior quantidade de indivíduos durante a lua crescente, contrariando os achados dos pesquisadores supra mencionados.

Coquillettidia venezuelensis normalmente é capturada com isca humana (FORATTINI et al. 1981) e em armadilha de Shannon (TEODORO et al. 1995). São mosquitos de hábito noturno, com acentuada atividade nas primeiras horas da noite, logo depois do crepúsculo vespertino. Essa atividade decresce gradativamente no 
decorrer da noite, mas no crepúsculo matutino é que se apresenta menos expressiva (ForATtinI et al. 1981; LOURENÇO-DE-OLIVEIRA \& SilVA 1985). Os resultados da presente investigação identificam-se com os dados dos supra citados autores, que no crepúsculo matutino das quatro fases lunares também encontraram diminuição do número e mesmo ausência de espécimes.
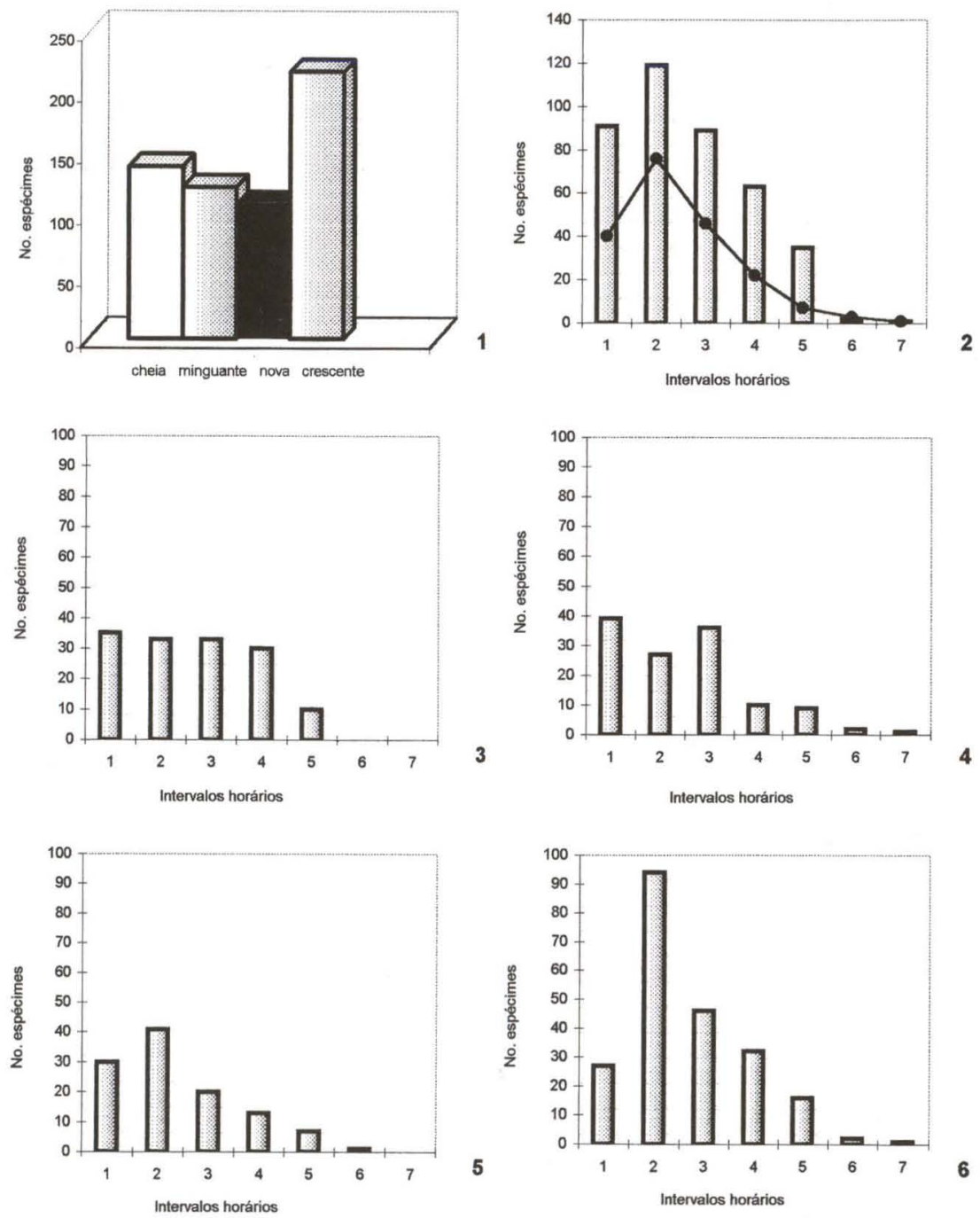

Figs 1-6. Total capturado de Coquillettidia venezuelensis em sete intervalos horários, Mata Atlântica, Morretes, Paraná, Brasil, outubro 1995 a janeiro 1996, em 48 noites nas luas: (1) cheia, minguante, nova, crescente; (2) nas quatro fases lunares: fêmeas (colunas), machos (linha); em 12 noites em cada fase lunar: (3) cheia; (4) minguante, (5) nova, (6) crescente. 
Tabela I. Total capturado de Coquillettidia venezuelensis em sete intervalos horários nas 48 noites das quatro fases lunares e as médias nos intervalos horários de temperatura ambiente, pluviosidade e umidade relativa do ar, Mata Atlântica, Serra do Marumbi, Morretes, Paraná, Brasil, de outubro de 1995 a janeiro de 1996.

\begin{tabular}{|c|c|c|c|c|c|c|}
\hline $\begin{array}{c}\text { Hora } \\
\text { de coleta }\end{array}$ & $\begin{array}{l}\text { Número de } \\
\text { fêmeas }\end{array}$ & $\begin{array}{l}\text { Número de } \\
\text { machos }\end{array}$ & Subtotal & $\begin{array}{l}\text { Temperatura } \\
\text { média }\left({ }^{\circ} \mathrm{C}\right)\end{array}$ & $\begin{array}{l}\text { Pluviosidade } \\
\text { média (mm) }\end{array}$ & $\begin{array}{c}\text { Umidade relativa } \\
\text { do ar }(\%)\end{array}$ \\
\hline \multicolumn{7}{|l|}{ Cheia } \\
\hline 1 & 24 & 11 & 35 & 22,2 & 0,08 & 90,7 \\
\hline 2 & 23 & 10 & 33 & 21,4 & 0,08 & 93,5 \\
\hline 3 & 31 & 2 & 33 & 20,5 & 0,16 & 95,2 \\
\hline 4 & 21 & 9 & 30 & 19,9 & 0,25 & 96,2 \\
\hline 5 & 7 & 3 & 10 & 19,6 & 0,16 & 96,7 \\
\hline 6 & 0 & 0 & 0 & 19,2 & 0,16 & 95,4 \\
\hline 7 & 0 & 0 & 0 & 21,1 & 0,16 & 93,6 \\
\hline Subtotal & 106 & 35 & 141 & 20,5 & 0,15 & 94,5 \\
\hline \multicolumn{7}{|l|}{ Minguante } \\
\hline 1 & 26 & 13 & 39 & 21,3 & 0,16 & 90,7 \\
\hline 2 & 20 & 7 & 27 & 20,5 & 0,00 & 93,5 \\
\hline 3 & 24 & 12 & 36 & 19,7 & 0,00 & 95,2 \\
\hline 4 & 8 & 2 & 10 & 19,1 & 0,08 & 96,2 \\
\hline 5 & 9 & 0 & 9 & 18,0 & 0,08 & 90,7 \\
\hline 6 & 1 & 1 & 2 & 15,9 & 0,08 & 95,4 \\
\hline 7 & 1 & 0 & 1 & 21,0 & 0,00 & 93,6 \\
\hline Subtotal & 89 & 35 & 124 & 19,3 & 0,07 & 94,5 \\
\hline \multicolumn{7}{|l|}{ Nova } \\
\hline 1 & 23 & 7 & 30 & 21,1 & 0,00 & 89,4 \\
\hline 2 & 33 & 8 & 41 & 20,2 & 0,00 & 91,0 \\
\hline 3 & 14 & 6 & 20 & 19,5 & 0,00 & 93,2 \\
\hline 4 & 12 & 1 & 13 & 18,9 & 0,00 & 94,3 \\
\hline 5 & 6 & 1 & 7 & 18,5 & 0,00 & 94,8 \\
\hline 6 & 0 & 1 & 1 & 18,2 & 0,00 & 95,1 \\
\hline 7 & 0 & 0 & 0 & 20,4 & 0,00 & 93,7 \\
\hline Subtotal & 88 & 24 & 112 & 19,5 & 0,00 & 93,1 \\
\hline \multicolumn{7}{|l|}{ Crescente } \\
\hline 1 & 18 & 9 & 27 & 20,2 & 0,25 & 97,0 \\
\hline 2 & 43 & 51 & 94 & 19,7 & 0,25 & 95,5 \\
\hline 3 & 20 & 25 & 45 & 19,2 & 0,08 & 96,1 \\
\hline 4 & 22 & 10 & 32 & 19,1 & 0,08 & 96,7 \\
\hline 5 & 13 & 3 & 16 & 18,9 & 0,50 & 97,0 \\
\hline 6 & 1 & 1 & 2 & 18,7 & 0,00 & 97,1 \\
\hline 7 & 0 & 1 & 1 & 20,4 & 0,08 & 96,1 \\
\hline Subtotal & 117 & 100 & 217 & 19,4 & 0,14 & 96,5 \\
\hline Total & 400 & 194 & 594 & - & - & - \\
\hline Porcentagem & 67,3 & 32,7 & 100,0 & - & - & - \\
\hline
\end{tabular}

Coquillettidia venezuelensis na lua cheia ocorreu nos intervalos horários de 1 a 5 e não ocorreu nos de 6 e 7, apresentando maior número de indivíduos no intervalo 1 (Tab. I, Fig. 3). Aplicando o teste de Friedman ANOVA, a hipótese Ho foi rejeitada. Realizando as comparações múltiplas, observou-se que para um nível de significância menor que $1,1 \%$ pode-se rejeitar Ho nas seguintes situações: entre intervalos horários 1 e 6,1 e 7, 2 e 6 e entre 2 e 7 . Aplicando o teste Wilcoxon Matched Pairs Test, a hipótese Ho foi rejeitada $(\mathrm{P}<0,0033)$, ou seja, as três primeiras horas diferem das quatro últimas. CHADEE (1992), porém, em Trinidad, coletou Anopheles (Kerteszia) bellator Dyar \& Knab, 1906 com isca humana emitindo flash 
de luz, durante as quatro fases da lua e verificou interação significativa entre as fases lunares e o horário de coleta de mosquitos. Observou ele um pico de ocorrência na lua cheia entre 16:00 e 20:00 horas. No entanto, BATES (1949) chama a atenção para a possibilidade de não haver correlação entre os períodos de atividade hematofágica e as outras atividades. Deste modo, o ritmo da atividade hematofágica pode ter perfil diferente de outras funções.

Tabela II. Total capturado de Coquillettidia venezuelensis em sete intervalos horários nas 48 noites das quatro fases lunares, Mata Atlântica, Serra do Marumbi, Morretes, Paraná, Brasil, de outubro de 1995 a janeiro de 1996.

\begin{tabular}{ccccccc}
\hline $\begin{array}{c}\text { Hora } \\
\text { de coleta }\end{array}$ & $\begin{array}{c}\text { Número de } \\
\text { mosquitos }\end{array}$ & $\begin{array}{c}\text { Número de } \\
\text { fêmeas }\end{array}$ & $\begin{array}{c}\text { Porcentagem } \\
(\%)\end{array}$ & $\begin{array}{c}\text { Número de } \\
\text { machos }\end{array}$ & $\begin{array}{c}\text { Porcentagem } \\
(\%)\end{array}$ & $\begin{array}{c}\text { Subtotal } \\
(\%)\end{array}$ \\
\hline 1 & 131 & 91 & 15,3 & 40 & 6,7 & 22,0 \\
2 & 195 & 119 & 20,0 & 76 & 12,8 & 32,8 \\
3 & 134 & 89 & 15,0 & 45 & 7,6 & 22,6 \\
4 & 85 & 63 & 10,6 & 72 & 3,7 & 7,3 \\
5 & 42 & 2 & 0,3 & 3 & 0,5 & 0,8 \\
6 & 5 & 1 & 0,2 & 1 & 0,2 & 0,4 \\
7 & 2 & 400 & 67,3 & 194 & 32,7 & 100,0 \\
\hline
\end{tabular}

$\mathrm{Na}$ lua minguante, Coquillettidia venezuelensis esteve presente em todos os intervalos horários (Tab. I, Fig. 4). Apresentou maior número de indivíduos nos intervalos 1 e 3. Aplicando o teste de Friedman ANOVA, rejeitou a hipótese Ho. Partindo para as comparações múltiplas, rejeitou-se Ho nas seguintes comparações: entre os intervalos horários 1 e 6 ao nível de significância de 4\%, entre 1 e 7 com um nível de significância de 3\%, e entre 3 e 6 ao nível de significância de $<1,1 \%$. Aplicando o teste de Wilcoxon Matched Pairs Test, a hipótese Ho foi rejeitada (P $<0,0044)$.

$\mathrm{Na}$ lua nova, a atividade de Coquillettidia venezuelensis foi semelhante ao da lua minguante com a diferença de ter apresentado maior número de indivíduos no intervalo horário 2 e ausência no 7 (Tab. I, Fig. 5). O teste de Friedman ANOVA, rejeitou a hipótese Ho $(\mathrm{P}<0,0000)$. Nas comparações múltiplas, rejeitou-se a hipótese Ho nas seguintes combinações: entre os intervalos 1 e 6 , a um nível de significância de $<1,1 \%$. Pelo teste de Wilcoxon Matched Pairs Test, a hipótese Ho foi rejeitada ( $\mathrm{P}<0,0067)$, ou seja, os três primeiros intervalos horários diferem dos quatro últimos. RUBio-PALIS (1992), em coletas de Anopheles (Nyssorhynchus) nuneztovari Gabaldon, 1940 e Hosfall (1943), em capturas de Anopheles (Anopheles) quadrimaculatus Say, 1824 obtiveram números mais expressivos de espécimes em lua nova. Entretanto, DEGALLIER et al. (1978), comparando os resultados de capturas horárias com isca humana com as feitas em armadilha luminosa em noites de lua cheia e lua nova, observaram que os dois tipos de captura de mosquitos podem ter rendimento semelhante, quando realizados durante a lua nova com espécies de hábitos diurnos e acentuadamente crepusculares, como é o caso de Coquillettidia venezuelensis, uma vez que têm o seu ciclo de atividade mais alterado pela condição da luminosidade do que as espécies mais ecléticas. 
Na lua crescente, Coquillettidia venezuelensis ocorreu em todos os intervalos horários, apresentando maior número de indivíduos no intervalo 2 à semelhança da lua nova. (Tab. I, Fig. 6). Aplicando o teste de Friedman ANOVA, a hipótese Ho foi rejeitada ( $\mathrm{P}<0,0001)$. Pelas comparações múltiplas, rejeitou-se a hipótese Ho nos seguintes combinados: entre os intervalos horários 2 e 6 , a um nível de significância de $<10 \%$, e entre os intervalos 2 e 7 , ao nível de significância de $<3 \%$. No teste de Wilcoxon Matched Pairs Test, a hipótese Ho foi rejeitada ( $\mathrm{P}<0,0051)$, ou seja, os três primeiros intervalos horários diferem dos quatro últimos. BIDLINGMAYER (1964), realizando capturas horárias de diferentes espécies de mosquitos com armadilhas sem isca, durante a noite, observou que a atividade de vôo está diretamente relacionada com a luminosidade. Entretanto, salienta o autor que, nas noites de lua crescente, os mosquitos voariam mais na primeira metade da noite, enquanto na lua minguante, na segunda. Na presente investigação, não se encontrou resultado semelhante, pois Coquillettidia venezuelensis foi sempre mais freqüente na primeira metade da noite, independentemente da fase da lua (Tab. I).

Dentre as variáveis climáticas avaliadas, pluviosidade, umidade relativa do ar e temperatura, somente esta última, oscilando em níveis mais elevados entre $22,2 \mathrm{e}$ $18,9^{\circ} \mathrm{C}$, no intervalo do crepúsculo vespertino até por volta de meia noite, pode ter influenciado na atividade do mosquito. É neste intervalo que a espécie ocorreu em maior abundância em todas as fases lunares (Tab. I), depois foi diminuindo paulatinamente até desaparecer de vez na madrugada e no crepúsculo matutino. Esta diminuição e até a ausência de mosquitos podem ter sido influenciadas pela queda da temperatura na segunda metade da noite, que oscilou de 18,0 a $15,9^{\circ} \mathrm{C}$ aliada ao aumento da luminosidade solar no crepúsculo matutino. A curva de regressão indicou a tendência do aumento do número de espécimes proporcionalmente à elevação da temperatura, até por volta de meia-noite. LOPES \& LOZOVEI (1996), em Londrina no Paraná, constataram a atividade noturna de Coquillettidia venezuelensis concentrar-se significativamente no crepúsculo vespertino. DEGALLIER et al. (1978), na Guiana Francesa, coletaram-na durante o dia todo, a espécie, porém, predominou à noite com pico após o pôr do sol. O fato de a sua atividade mais intensa restringir-se ao intervalo do crepúsculo vespertino até próximo da meia-noite, durante todo o ciclo lunar na presente pesquisa, leva à conclusão de isto constituir sua característica, obedecendo a critérios fisiológicos e exigências bionômicas específicas no habitat estudado.

AGRADECIMENTOS. Aos Professores Ricardo Sandes Ehlers e Joel Maurício Correia da Rosa, Departamento de Estatística, Universidade Federal do Paraná, pelo auxilio na elaboração conjunta dos testes estatísticos.

\section{REFERÊNCIAS BIBLIOGRÁFICAS}

Allen, S.A.; J.F. Day \& J.D. Edman. 1987. Visual ecology of biting flies. Ann. Rev. Ent. 32: 297-316. DHN. 1995. Almanaque náutico. Rio de Janeiro, Ministério da Marinha, Diretoria de Hidrografia e Navegação. XXXV+287p.

. 1996. Almanaque náutico. Rio de Janeiro, Ministério da Marinha, Diretoria de Hidrografia e Navegação, XXXV+287p.

BAtes, M. 1949. The Natural History of Mosquitoes. Macmillan Company, New York, 379p.

Revta bras. Zool. 18 (2): 365 - 374, 2001 
BIDLINGMAYER, W.L. 1964. The effect of moonlight on the flight activity of mosquitoes. Ecology 45: 87-94.

1974. The influence of environmental factors and physiological stage on flight patterns of mosquitoes taken in the vehicle aspirator and truck suction bait and new jersey light traps. Jour. Med. Ent. 11: 119-146.

BOWDEN, J. 1973. The significance of moonlight in photoperiodic responses of insects. Bull. Ent. Res. 62: 605-612.

Bradley, G.H. \& T.E. MCNeEl. 1935. Mosquito collections in Florida with the new jersey light trap. Jour. Econ. Ent. 28: 780-786.

CHADEE, D.D. 1992. Indoor and outdoor host-seeking rhytms of Anopheles bellator (Diptera: Culicidae) in Trinidad, West Indies. Jour. Med. Ent. 29 (3): 567-569.

Charlwood, J.D.; R. PARU; H. DaGoro \& M. LAGOG. 1986. Influence of moonlight and gonotrophic age on biting activity of Anopheles farauti (Diptera: Culicidae) from Papua New Guinea. Jour. Med. Ent. 23: 132-135.

Consoli, R.G.B. \& R. Lourenço-DE-OliveIra. 1994. Principais Mosquitos de Importância Sanitária no Brasil. Rio de Janeiro, Fiocruz, 225p.

DAvies, J.B. 1975. Moonlight and the biting activity of Culex (Melanoconion) portesi Senevet and Abonnenc and Culex (Melanoconion) taeniopus Dyar \& Knab (Diptera: Culicidae) in Trinidad forests. Bull. Ent. Res. 65: 81-96.

Degallier, N.; F.X. Pajot; R. Kramer; J. Claustre; S. Bellony \& F. Le pont. 1978. Rhytmes d'activité des culicidés de la Guyane Française (Diptera: Culicidae). Cah. ORSTOM., Sér. Ent. Med. Parasitol. 16: 73-84.

Foley, D.H.; A. Barnes \& J.H. Bryan. 1992. Anopheles annulipes Walker (Diptera: Culicidae) at griffith, New-South-Wales. 4. Phenology of 2 sibling species. Jour. Aust. ent. Soc. 31: 91-96.

Forattini, O. P. 1965a. Entomologia Médica. São Paulo, Universidade de São Paulo, Vol. 2, 506 p.

- 1965b. Entomologia Médica. São Paulo, Fac. Saúde Pública, Univ. de São Paulo, Vol. 3, $416 \mathrm{p}$.

Forattini, O.P.; A.C. Gomes; J.L. F. Santos; E.A.B. Gal.ati; E.X. Rabello \& D. Natal. 1981. Observações sobre atividade de mosquitos Culicidae, em mata residual no Vale do Ribeira, São Paulo, Brasil. Revta Saúde Públ. 15: 557-586.

Hervé, J.P.; N. Degallier; A.P.A. Travassos da Roda; F.P. Pinheiro \& G.C. Sá Filho. 1986. Instituto Evandro Chagas - $\mathbf{5 0}$ anos de contribuição às Ciências Biológicas e à Medicina Tropical. Belém, Fund. Serv. Saúde Pública, Vol. 1, 529p.

Hosfall, W.R. 1943. Some responses of the malaria mosquito to light. Ann. Ent. Soc. Amer. 36 (1): 41-45.

LOPES, J. \& A.L. Lozover. 1996. Ecologia de mosquitos (Diptera, Culicidae) em criadouros naturais e artificiais de área rural do norte do Paraná, Brasil. II. Coletas com isca humana. Revta bras. Zool. 13 (3): 585-596.

Lourenço-de-Oliveira, R. \& T.F. Silvı. 1985. Alguns aspectos da ecologia de mosquitos (Diptera, Culicidae) de uma área de planície (Granjas Calábria), em Jacarepaguá, Rio de Janeiro. II. Freqüência mensal e no ciclo lunar. Mem. Inst. Oswaldo Cruz 80 (2): 123-133.

Mishira, A.K.; C.F. CuRTiS \& V.P. Sharma. 1996. Influence of moonlight on light-trap catches of the malaria vector Anopheles culicifacies (Diptera: Culicidae) in central India. Bull. Ent. Res. 86: 475-479.

PRATT, H.J. 1948. Influence of the moon on light trap collections of Anopheles albimanus in Puerto Rico. Jour. Nat. Malar. Soc. 7: 212-220.

Provost, M.W. 1959. The influence of moolight on light-trap catches of mosquitoes. Ann. Ent. Soc. Amer. 52: 261-171.

REINERT, J.F. 1975. Mosquito generic and subgeneric abbreviations (Diptera: Culicidae). Mosq. Systematics 7 (2): 105-110.

RUBio-PALIS, Y. 1992. Influence of moolight on light trap catches of the malaria vector Anopheles 
nuneztovari in Venezuela. Jour. Amer. Mosq. Control. Assoc. 8 (2): 178-180.

Siegel, S. 1975. Estatística não Paramétrica. Rio de Janeiro, McGraw-Hill, 333p.

Singh, N.; A.K. Mishra; C.F. CuRTIS \& V.P. Sharma. 1996. Influence on moonlight trap catches of the malaria vector Anopheles culicifacies (Diptera, Culicidae) in Central India. Bull. Ent. Res. 86 (4): 475-479.

Teodoro, U.; A.L.F. Guilherme; A.L. Lozovei; V. la Salvia Filho; Y. Fukushigue; R.P. Spinosa; M.E.M.C. Ferreira; O.C. BARbosa \& E.M. Lima. 1995. Culicídeos do lago de Itaipu, no Rio Paraná, sul do Brasil. Revta Saúde Públ. 29 (1): 6-14.

Veloso, H.P.; A.L.R. Rangel \& J.C.A. Lima. 1991. Classificação da Vegetação Brasileira adaptada a um Sistema Universal. Rio de Janeiro, IBGE, 123p.

Recebido em 11.V.2000; aceito em 02.V.2001. 